\title{
Cavernomas pontinos extraventriculares. Parte I. Acesso transtentorial a cavernoma pontino dorsolateral
}

\author{
Marcos Augusto Stávale Joaquim', Gustavo Cartaxo Patriota², André de Macedo Bianco ${ }^{3}$ \\ Instituto de Neurociências, São Paulo. \\ Serviço de Neurocirurgia do Hospital 9 de Julho, São Paulo.
}

\section{RESUMO}

Objetivo: Descrever a via transtentorial, por meio de craniotomia têmporo-occipital, para remoção de cavernoma pontino de localização dorsolateral, exemplificado pela apresentação de um caso. Conclusão: $O$ acesso transtentorial demonstra-se útil e seguro para remoção de lesões localizadas nessa região da ponte.

PALAVRAS-CHAVE

Cavernoma. Angioma cavernoso. Cirurgia do tronco cerebral.

\section{ABSTRACT}

Extraventricular pontine cavernomas. Part I. Transtentorial route to remove a postero-lateral pontine cavernoma

Objective: To present the transtentorial route through a temporal-occipital craniotomy for surgical removal of a posterior-lateral pontine cavernomas, exemplified by the presentation of a case. Regional anatomy is discussed. Conclusion: The transtentorial approach may be used safely to remove lesions of highly located in the posterior-lateral region of the pons.

\section{KEY WORDS}

Cavernoma. Cavernous angioma. Brain stem surgery.

\section{Introdução}

Os cavernomas provocam hemorragias de diferentes intensidades ao longo de sua existência. Hemorragias em áreas eloquentes ou áreas de grande densidade e concentração de fibras e núcleos, como o tronco corticoencefálico, podem produzir sequelas irreparáveis s $^{2-4,6,8,10,14,20-24}$. Os cavernomas devem ser removidos profilaticamente quando o risco da cirurgia for menor do que o risco da história natural da doença. A grande maioria dos cavernomas deve ser removida.

As lesões do tronco encefálico foram consideradas inoperáveis no passado. A evolução da microcirurgia, dos métodos auxiliares de ressecção, da cirurgia guiada por imagens e das técnicas de monitorização neurofisiológica permite atualmente que essas lesões sejam submetidas ao tratamento cirúrgico. Os acessos ao tronco encefálico citados em publicações anteriores não incluem a citação do acesso transtentorial póstero-lateral, exceto as publicações de Ono e cols., ${ }^{18}$ Kashimura e cols. ${ }^{19}$ e Kumabe e cols. ${ }^{11}$

\section{Cirurgia dos cavernomas do tronco encefálico}

Para o planejamento da cirurgia no tronco encefálico, deve-se ter conhecimento segmentar das relações neurovasculares (anatomia topográfica externa), assim como

1 Neurocirurgião do Instituto de Neurociências, São Paulo, SP, Brasil. Doutor em Medicina pela Faculdade de Medicina da Universidade de São Paulo (FMUSP).

2 Neurocirurgião do Instituto de Neurociências, São Paulo, SP, Brasil.

3 Neurocirurgião do Serviço de Neurocirurgia do Hospital 9 de Julho, São Paulo. 
da disposição dos feixes de fibras e núcleos que ali se localizam (anatomia topográfica interna). Tais disposições devem estar memorizadas pelo cirurgião e determinam o tipo de monitorização a ser utilizado., $4,12,13,16,18$

Na região dessa lesão pontina, em relação à topografia externa, nota-se superiormente o colículo inferior e seu braço, a veia basal de Rosenthal e as artérias cerebrais posteriores e cerebelar superior e seus ramos. ${ }^{7}$ Podem ser vistas veias tectais. Anteriormente, há o sulco das veias mesencefálicas laterais e, inferiormente, visualiza-se o lobo quadrangular do cerebelo que, quando retraído inferiormente, expõe a fissura cerebelo-mesencefálica (fissura pré-centro cerebelar) e o sulco pontomesencefálico. ${ }^{18} \mathrm{Em}$ relação à anatomia vascular e dos troncos nervosos, além do nervo troclear, inferiormente localiza-se o nervo trigêmeo, emergindo da transição entre a ponte e o pedúnculo cerebelar médio. Tais estruturas citadas neste parágrafo situam-se nos espaços incisurais posterior e médio, ${ }^{18}$ encontrados estudando-se a borda tentorial. A cisterna quadrigeminal continua-se com a cisterna cerebelomesencefálica. A cisterna ao redor do mesencéfalo (cisterna perimesencefálica) corresponde às asas da cisterna ambiens. Esta possui um compartimento supratentorial e um infratentorial que contém o nervo troclear. ${ }^{15,25}$

Em relação à topografia interna, na ponte deve ser conhecida a disposição das fibras longitudinais, transversas e dos núcleos pontinos. Nessa região são importantes o lemnisco lateral e seu núcleo, o lemnisco espinhal e lemnisco medial, e, mais anteriormente, o trato corticoespinal, corticopontino e corticonuclear, além das fibras transversas e núcleos pontinos. Os núcleos trigeminais e as fibras do pedúnculo cerebelar superior estão relacionados.

Cavernomas do tronco encefálico frequentemente revelam uma coloração amarelada na superfície, consequente a hemorragias prévias. Nem sempre o centro dessa área é o melhor acesso à lesão e a anatomia funcional local deve ser considerada em conjunto com a navegação. Após a intrusão no tronco encefálico, o cavernoma é visualizado, dissecado e removido. A coagulação bipolar fraca pode diminuir a lesão e facilitar a remoção. $O$ tecido neural normal não deve ser empurrado ou manipulado. A área gliótica circunjacente, com depósito de hemossiderina, não deve ser removida, pois lesões serão provocadas e, nesse local, não há risco de epilepsia pelo depósito de ferro. Os angiomas venosos locais devem ser preservados. Movimentos leves são realizados e o cirurgião deve interrogar continuamente o neurofisiologista durante a manipulação leve e antes das remoções, para que o neurofisiologista possa ajudar a impedir que a lesão ocorra, e não avisar que ela já ocorreu. Para tanto, o cirurgião deve interrogar o monitoramento a cada compressão ou deslocamento tecidual leve. Tal conduta aumenta o tempo da operação, requer paciência, mas é fundamental na cirurgia do tronco encefálico. $3,5,6,9,14,17,21-24$

\section{Particularidades do acesso em questão}

Uma craniotomia têmporo-occipital de base larga é planejada com guia estereotáxico ou com neuronavegação. A informatização do acesso é indispensável. A craniotomia de base larga permite manipulação angular dos instrumentos ao longo da borda do forâmen de Pacchioni (espaço incisural) e a observação e o isolamento da veia de Labbé a ser preservada. A membrana aracnoide do espaço incisural deve ser aberta e seu conteúdo visualizado. Em seguida, os seios venosos tentoriais são coagulados e a tenda do cerebelo é aberta até a sua borda. ${ }^{1}$ Esse procedimento é guiado pelo planejamento informatizado, em direção às asas da cisterna.

\section{Caso-exemplo}

BG, 41 anos, sexo feminino. Admitida no prontoatendimento com queixa de síndrome vestibular aguda e cefaleia. Ao exame neurológico, havia um quadro vestibular central, sem diplopia ou alterações de outros nervos cranianos. A tomografia computadorizada demonstrou uma hemorragia localizada e recente, situada na porção dorsal, lateral e superior da ponte, logo abaixo do sulco pontomesencefálico. A ressonância magnética confirmou o achado (Figuras 1 a 4). A cirurgia foi realizada com a remoção completa da lesão e o exame neurológico da paciente no pós-operatório foi normal (Figuras 5 a 7). A monitorização neurofisiológica intraoperatória revelou descargas por estimulação mecânica piramidal no membro inferior contralateral durante a dissecção da lesão lateralmente ao trato piramidal.

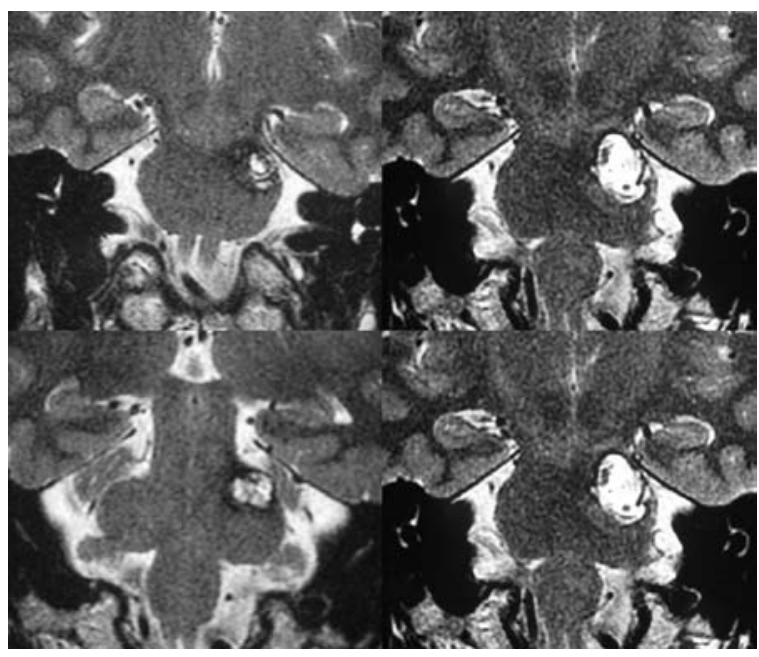

Figura 1-Ressonância magnética, sequência T2, mostrando a lesão pontina. 


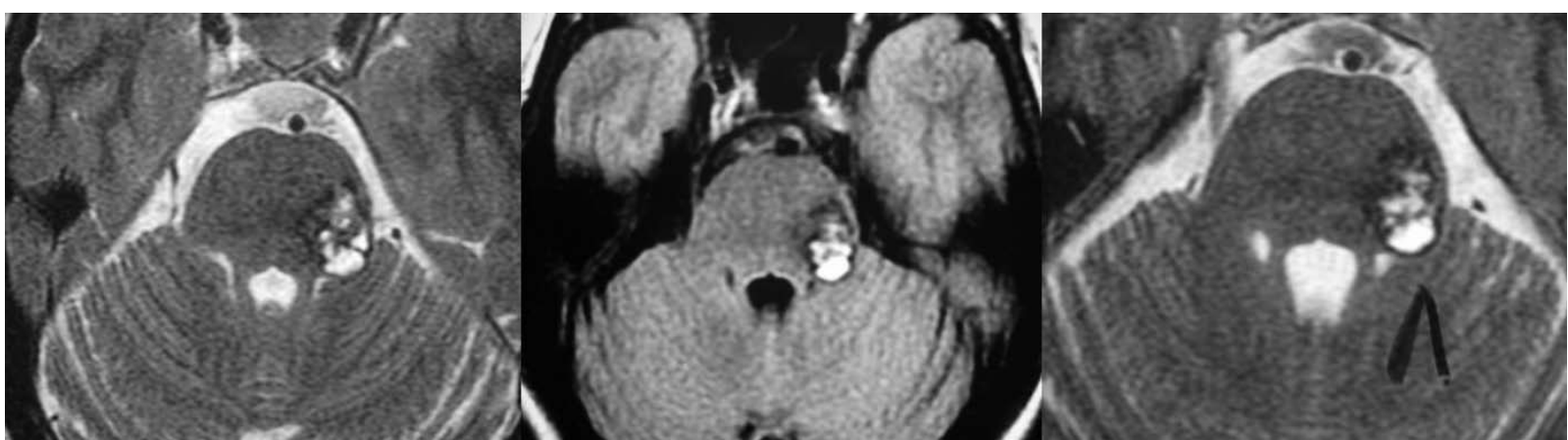

Figura 2 - Ressonância magnética, cortes axiais, e relação topográfica com a fissura cerebelo-pontina.

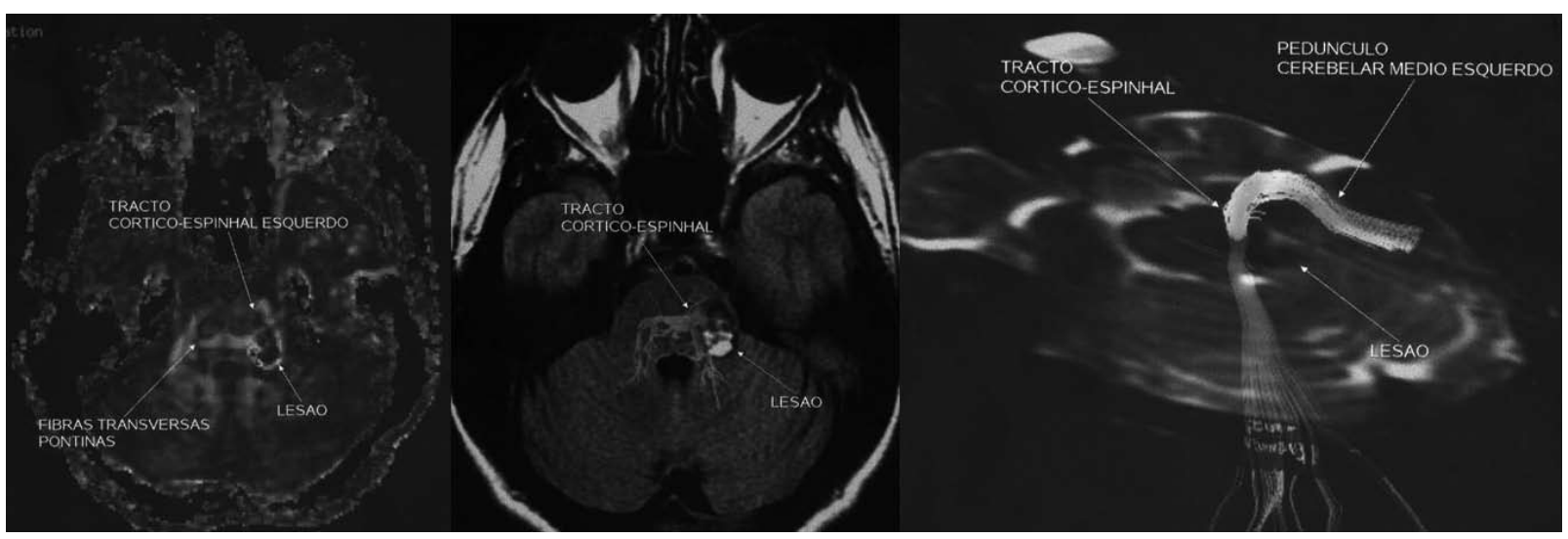

Figura 3 - Tratografia corticoespinal e das fibras do pedúnculo cerebelar médio. Relações nucleares podem ser inferidas.

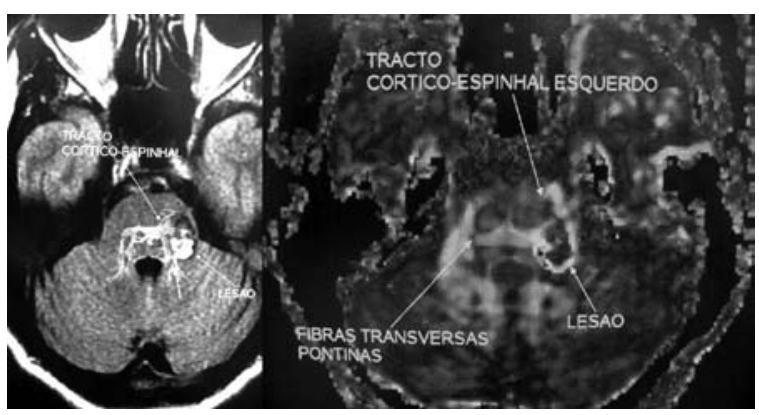

Figura 4-Relações com o trato corticoespinal.

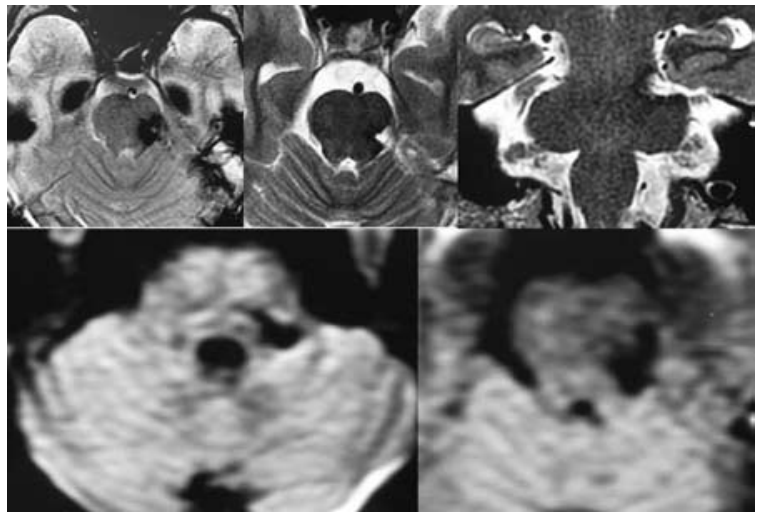

Figura 6 - Ressonância magnética. Controle pós-operatório.

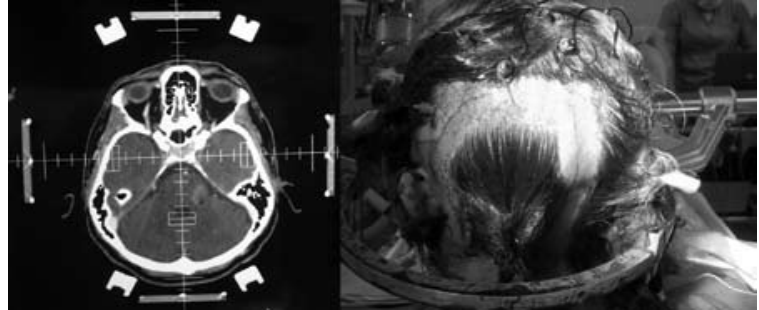

Figura 5-Mapeamento estereotáxico.

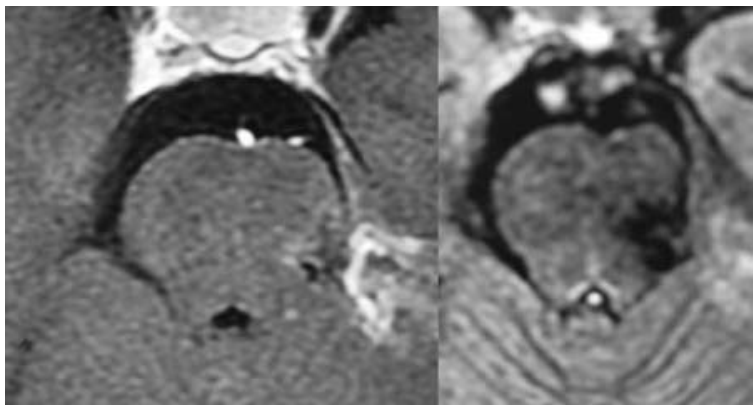

Figura 7 - Ressonância magnética. Controle pós-operatório. 


\section{Discussão}

A cirurgia do tronco encefálico tornou-se, com boa frequência, um procedimento de baixo risco, mas exige tecnologia elaborada. As lesões com riscos de hemorragias devem ser removidas para que se evitem danos futuros. Os cavernomas do tronco encefálico são retirados atualmente com frequência comparável à dos cavernomas supratentoriais.

\section{Conclusão}

As lesões em localizações incomuns do tronco encefálico merecem publicações para a contribuição na tomada de decisões nos casos semelhantes. $\mathrm{O}$ acesso a essa região pontina merece essa atenção. Os cavernomas pontinos craniais e laterais, situados logo abaixo do sulco pontomesencefálico podem ser removidos com segurança por esse acesso.

\section{Referências}

1. Browder J, Kaplan HA, Krieger AJ. Anatomical features of the straight sinus and its tributaries. J Neurosurg. 1976;44: 55-61.

2. Conway JE, Rigamonti D. Cavernous malformations: a review and current controversies. Neurosurg Q. 2001;16:15-23.

3. Endo S, Matsumura N, Kurimoto M, Takaku A. Surgically ressected brain stem cavernous angioma in an infant. Childs Nerv Syst. 1997;13:613-5.

4. Fox JR. Tentorial section for decompression of the brain stem and large basilar aneurysm. Case Report. J Neurosurg. 1968;28:74-7.

5. Fritschi JA, Reulen HJ, Spetzler RF, Zabramski JM. Cavernous malformations of the brain stem. A review of 139 cases. Acta Neurochir (Wien). 1994;130:35-46.

6. Haque R, Kellner CP, Solomon RA. Cavernous malformations of the brain stem. Clin Neurosurg. 2008;55:88-96.

7. Hardy DG, Rhoton AL. Microcirurgical relationships of the superior cerebelar artery and trigeminal nerve. J Neurosurg. 1983;59:63-105.

8. Hauch EF, Barnett SL, White JA, Samson D. Symptomatic brainstem cavernomas. Neurosurgery. 2009;64:61-71.

9. Kashimura H, Inoue T, Ogasawara K, Ogawa A. Pontine cavernous angioma resected using the subtemporal, anterior transpetrosal approach determined using threedimensional anisotropy contrast imaging: technical case report. Neurosurgery. 2006;58(1 Suppl):ONS-E175.
10. Kondziolka D, Lundsford LD, Kestle JR. The natural history of cerebral cavernous malformations. J Neurosurg. 1995;83:820-4.

11. Kumabe T, Suzuki M, Yoshimoto T, Suzuki J. A case of cavernous angioma extend from the ventral part of the pons to the midbrain: subtemporal and trans-tentorial approach. No Shinkei Geka. 1988;16:1193-7.

12. Martin RG, Grant JL, Peace D, Theiss C, Rhoton AL. Microsurgical relationships of the anterior inferior cerebelar artery and the facial vestíbulo cochlear nerve complex. Neurosurgery. 1980;6:483-507.

13. Martinez AG, Oliveira E, Tedeschi H, Wen HT, Rhoton AL. Microsurgical anatomy of the brain stem. Oper Tech Neurosurg. 2000;3:80-6.

14. Mathiesen T, Edner G, Kihlstrom L. Deep and brainstem cavernomas: a consecutive 8-year series. J Neurosurg. 2003;99:31-7.

15. Matsuno M, Rhoton AL, Peace DP. Microcirurgical anatomy of the posterior fossa cisterns. Neurosurgery. 1988;23:58-80.

16. Matsushima T, Rhoton AL, Oliveira E, Peace D. Microsurgical anatomy of the veins of the posterior fossa. J Neurosurg. 1983;59:63-105.

17. Okuno S, Nishi N, Hirabayashi H, Sakaki T. A surgical case of growing cavernous angioma at the pontomedullary junction. No Shinkei Geka. 2000;28:891-7.

18. Ono M, Rhoton AL, Barry M. Microcirurgical anatomy of the region of the tentorial incisura. J Neurosurg. 1984;60: 365-99.

19. Porter RW, Detwiler PW, Spetzler RF, Lawton MT, Baskin J, Derksen PT, et al. Cavernous malformations of the brainstem: experience with 100 patients. J Neurosurg. 1999;90:50-8.

20. Rodinson JR, Awad IA, Little JR. Natural history of the cavernous angioma. J Neurosurg. 1991;75:709-14.

21. Saito N, Sasaki T, Chikui E, Yuyama R, Kirino T. Anterior transpetrosal approach for pontine cavernous angioma: case report. Neurol Med Chir (Tokyo). 2002;42:272-4.

22. Sami M, Eghbal R, Carvalho GA, Matthies C. Surgical management of brainstem cavernomas. J Neurosurg. 2001;95:825-32.

23. Sandalcioglu IE, Wiedemayer H, Secer S, Asgari S, Stolke D. Surgical removal of brain stem cavernous malformations: surgical indications, technical considerations, and results. J Neurol Neurosurg Psychiatry. 2002;72:351-5.

24. Symon K, Jackowski A, Bills D. Surgical treatment of pontomedullary cavernomas. Br J Neurosurg. 1991;5:339-47.

25. Yasargil MG, Kasdaglis K, Jain KK, Weber HP. Anatomical observations of the subarachnoid cisterns of the brain during surgery. J Neurosurg. 1976;44:298-302.

Original recebido em junho de 2008

Aceito para publicação em março de 2009

Endereço para correspondência

Marcos Augusto Stávale Joaquim

Alameda Campinas, $1.360,16^{\circ}$ andar

01404-002 - São Paulo, SP, Brasil

E-mail:marcos.stavale@terra.com.br 